\title{
Noble gas cluster ions
}

\author{
Yunus Kaya ${ }^{1, a}$, Yalçın Kalkan², and Rob Veenhof ${ }^{2,3}$ \\ ${ }^{1}$ Department of Chemistry, Faculty of Arts and Sciences, Uludağ University, 16059 Bursa, Turkey. \\ ${ }^{2}$ Department of Physics, Faculty of Arts and Sciences, Uludağ University, 16059 Bursa, Turkey. \\ ${ }^{3}$ RD51, CERN.
}

\begin{abstract}
In this work, a reaction mechanism of formation of noble gas $(\mathrm{Ng})$ cluster ions has been theoretically investigated in detail. The kinetic studies of formation of $\mathrm{Xe}^{+} \mathrm{Xe}$ cluster in $\mathrm{Xe}, \mathrm{Ar}^{+} \mathrm{Ar}$ cluster ions in $\mathrm{Ar}$, and $\mathrm{Ne}^{+} \mathrm{Ne}$ cluster ions in $\mathrm{Ne}$ have been made as theoretically. The optimized structures in the ground state were calculated using the density functional theory (DFT) by the B3LYP method combined with the Stuttgart/Dresden effective core potential basis set (SDD). In addition, we calculated the rate constants of all cluster formations. The results are $1.15 \times 10^{-31}, 3.58 \times 10^{-31}, 0.23 \times 10^{-31} \mathrm{~cm}^{6} / \mathrm{s}$, respectively for Neon, Argon, Xenon cluster ions.
\end{abstract}

\section{Introduction}

A general classification of clusters according to their binding strength into van der Waals clusters, molecular clusters, hydrogen bonded clusters, ionic clusters, valence clusters and metal clusters has been proposed by Jortner [1]. The current paper focuses on small aggregates, which are bound by weak van der Waals forces in the ionic state. Here, we consider the dimers with one van der Waals bond as the smallest cluster entities.

In recent decades, quantum chemistry calculations have been used for various chemical fields such as reaction pathway analysis and spectroscopic assignments due to the theoretical developments, especially accuracy improvement of functional in density functional theory (DFT), and high-speed parallel computers.

The kinetic studies of formation Noble gas $(\mathrm{Ng})$ cluster ions, which are $\mathrm{Xe}^{+} \mathrm{Xe}$ cluster in $\mathrm{Xe}, \mathrm{Ar}^{+} \mathrm{Ar}$ cluster ions in $\mathrm{Ar}$, and $\mathrm{Ne}^{+} \mathrm{Ne}$ cluster ions in $\mathrm{Ne}$ have not been found as experimentally or theoretically in literature. Therefore, in this study, we report the DFT-based mechanistic studies and spectroscopic analyses on formations of these clusters. In addition, we have calculated the rate constants all cluster formations.

\section{Computational methods}

The experiments and their analysis described in the literature [2] are complemented by quantum chemical calculations. These calculations concern the equilibrium structures of the relevant clusters, their vibration frequencies and rotational constants. All calculations were conducted using density functional theory (DFT) as implemented in the GAUSSIAN 09 program package [3]. The molecular geometries and vibrational frequencies of the

\footnotetext{
ae-mail: ykaya@uludag.edu.tr
}

all molecules in the ground state have been calculated using the B3LYP [4] with the SDD basis set [5]. Vibrational frequencies were used for calculation of the Gibbs free energies.

\section{Result and discussion}

In this study, a mechanism of the noble gas cluster formation has been theoretically investigated in detail. Firstly, the mechanism of the formation of cluster ions was suggested for the noble gaseous. Then, all suggested geometries were optimized by DFT/B3LYP method and SDD level. The optimized geometries are shown in figure 1 . The Gibbs free and the relative energies for the formation reactions were calculated at same basis set in atmospheric pressure and at room temperature.
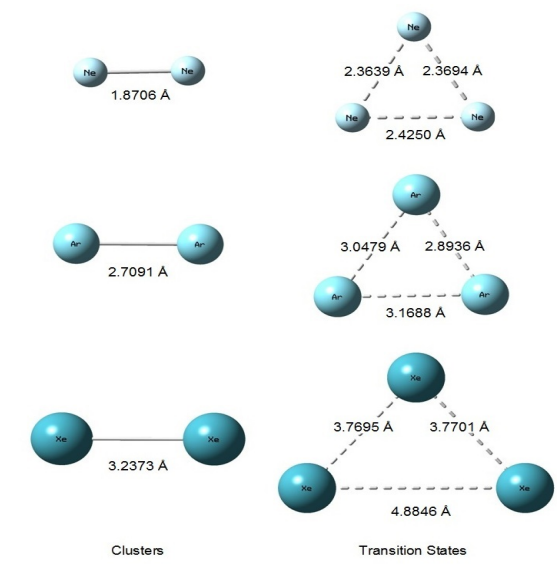

Figure 1. Optimized geometries of cluster ions and TS for Ng.

The $\mathrm{Ng}$ cluster ions can be formed in a three-body interaction at atmospheric pressure. The mechanism of the formation reaction of the $\mathrm{Ng}$ cluster ions involves one 
step, which are composed of triangular plane over transition state (TS).

According to the transition state theory, the rate constants are related to activation Gibbs free energies $\left(\Delta G^{*}\right)$. Therefore, analysis of these quantities in kinetic is of special importance. The activation Gibbs free energies related to TS are 249.6, 247.0 and $253.9 \mathrm{~kJ} / \mathrm{mole}$, respectively for Neon, Argon and Xenon cluster ions (Table 1).

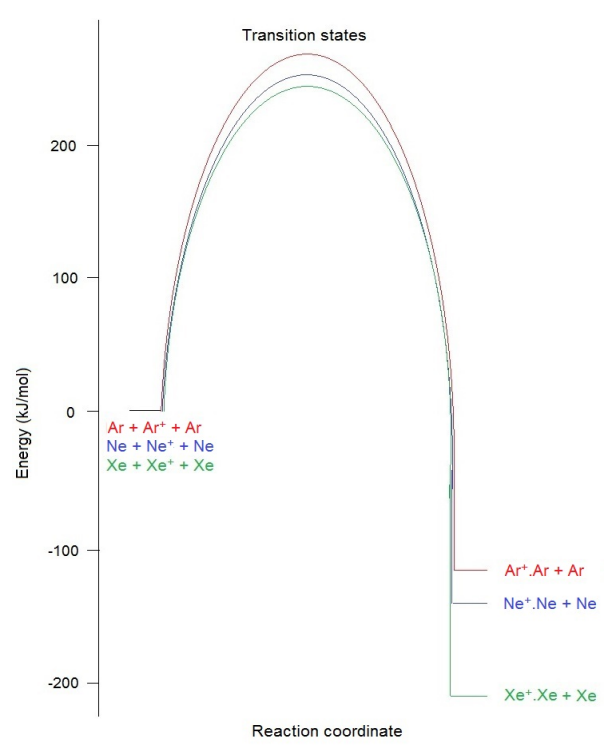

Figure 2. Enery profile for the formation of cluster ions of $\mathrm{Ng}$.

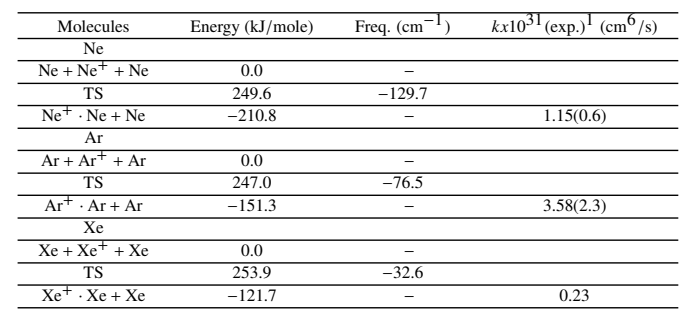

Table 1. Relative energy, negative frequency and rate constants of the structures of the reaction paths for formation of clusters ions for $\mathrm{Ng}$.

Using traditional transition state theory:

$$
k=\frac{k_{\mathrm{b}} T}{h} \exp \left[-\frac{\Delta \mathrm{G}^{*}}{\mathrm{RT}}\right]
$$

where $k_{\mathrm{b}}, h, \mathrm{R}$, and $\mathrm{T}$ are the Boltzmann constant, Plank constant, gas constant, and temperature, respec- tively. The values of theoretical rate constants are calculated from equation 1 , which are equal to $k=1.15 \times 10^{-31}$, $3.58 \times 10^{-31}, 0.23 \times 10^{-31} \mathrm{~cm}^{6} / \mathrm{s}$, respectively for Neon, Argon, Xenon cluster ions. The experimental values are $0.6 \times 10^{-31}$ and $2.3 \times 10^{-31} \mathrm{~cm}^{6} / \mathrm{s}$ for neon and argon cluster ions, respectively [2]. The calculated rate constants are in good agreement with experimental values. These all calculations show that the relative energy of TS for formation of argon cluster ion is smaller than TS for formation of neon cluster ion. Therefore, it was determined that the formation of argon cluster ion is faster than formation of neon cluster ion, although the neon is smaller than Argon [6]. The reason is that the cross section of argon may be higher than neon. In addition, the smallest rate constant was calculated in the formation of Xenon cluster ions. It is thought that the atomic size may be more effective than the cross section in the formation of cluster ions.

\section{Conclusion}

In this study, the kinetic studies of formation of $\mathrm{Ng}$ cluster ions have been calculated using density functional theory (DFT). The values of theoretical rate constants are calculated at $1.15 \times 10^{-31}, 3.58 \times 10^{-31}, 0.23 \times 10^{-31} \mathrm{~cm}^{6} / \mathrm{s}$, respectively for Neon, Argon, Xenon cluster ions.

\section{Acknowledgments}

This work is supported by the Scientific Research Projects Unit of Uludağ University, project number: KUAP(F)2015/20.

\section{References}

[1] Jortner, J., Bunsenges, B., Phys. Chem. 88188 (1984).

[2] Kalkan, Y., Arslandok, M., Cortez, A.F.V., Kaya, Y., Tapan, I., Veenhof, R., JINST., DOI: 10.1088/17480221/10/07/P07004.

[3] Frisch, M.J., et al., Gaussian 03, Revision E.01, Gaussian, Inc., Wallingford, CT, (2004).

[4] Becke, A.D., J. Chem. Phys. 98 5648-5652, (1993).

[5] Lv G., Wei F., Li Q., Shen Q., Jiang H., Zhou Y., Wang X. J. Nanosci. Nanotechnol. 10, 809-818 (2010).

[6] Smirnov, B. M., Cluster Ions and Van der Waals Molecules, Gordon and Breach Science Publishers (1992). 